Article

\title{
Predictors of Clinical Outcome in Women with Pelvic Organ Prolapse Who Underwent Transvaginal Mesh Reconstruction Surgery
}

\author{
Ting-Hsuan Lin ${ }^{1}$, Fung-Chao Tu ${ }^{1}$, Ho-Hsiung Lin ${ }^{1,2}$ and Sheng-Mou Hsiao ${ }^{1,2,3, *(D)}$ \\ 1 Department of Obstetrics and Gynecology, Far Eastern Memorial Hospital, Banqiao, \\ New Taipei 220409, Taiwan; lindonut713@gmail.com (T.-H.L.); fctu77@yahoo.com.tw (F.-C.T.); \\ hhlin@ntuh.gov.tw (H.-H.L.) \\ 2 Department of Obstetrics and Gynecology, National Taiwan University College of Medicine and the Hospital, \\ Taipei 100225, Taiwan \\ 3 Graduate School of Biotechnology and Bioengineering, Yuan Ze University, Taoyuan 320315, Taiwan \\ * Correspondence: smhsiao2@gmail.com; Tel.: +886-2-89667000 (ext. 1424); Fax: +886-2-89665567
}

Citation: Lin, T.-H.; Tu, F.-C.; Lin H.-H.; Hsiao, S.-M. Predictors of Clinical Outcome in Women with Pelvic Organ Prolapse Who Underwent Transvaginal Mesh Reconstruction Surgery. Medicina 2022, 58, 148. https://doi.org/ 10.3390/medicina58020148 Academic Editor: Marco Torella Received: 9 November 2021 Accepted: 17 January 2022 Published: 19 January 2022

Publisher's Note: MDPI stays neutral with regard to jurisdictional claims in published maps and institutional affiliations.

Copyright: (C) 2022 by the authors. Licensee MDPI, Basel, Switzerland. This article is an open access article distributed under the terms and conditions of the Creative Commons Attribution (CC BY) license (https:/ / creativecommons.org/licenses/by/ $4.0 /)$.

\begin{abstract}
Background and Objectives: To identify the predictors of clinical outcomes in women with pelvic organ prolapse (POP) who underwent transvaginal reconstruction surgery, especially with transobturator mesh fixation or sacrospinous mesh fixation. Materials and Methods: All women with POP who underwent transvaginal reconstruction surgery, especially with transobturator mesh fixation or sacrospinous mesh fixation, were reviewed. Results: Between January 2011 and May 2019, a total of 206 consecutive women were reviewed, including 68 women receiving POP reconstruction with transobturator mesh fixation and 138 women who underwent POP reconstruction with sacrospinous mesh fixation. The least experienced surgeon (hazard ratio $=804.6$ ) and advanced stage of cystocele (hazard ratio $=8.80$ ) were the predictors of POP recurrence, especially those women with stage 4 of cystocele. Young age (hazard ratio $=0.94$ ) was a predictor for mesh extrusion, especially those women with age $\leq 67$ years. Follow-up interval (odds ratio $=1.03, p=0.02$ ) was also an independent predictor of mesh extrusion. High maximum flow rate $(\mathrm{Qmax}$, hazard ratio $=1.03)$ was the sole predictor of postoperative stress urinary incontinence, especially those women with Qmax $\geq 19.2 \mathrm{~mL} / \mathrm{s}$. Preoperative overactive bladder syndrome (hazard ratio $=3.22$ ) were a predictor for postoperative overactive bladder syndrome. In addition, overactive bladder syndrome rate improved after surgery in the sacrospinous group ( $p=0.0001)$. Voiding dysfunction rates improved after surgery in both sacrospinous and transobturator groups. Conclusions: Predictors of clinical outcome in women who underwent transvaginal POP mesh reconstruction are identified. The findings can serve as a guide for preoperative consultation of similar procedures.
\end{abstract}

Keywords: pelvic organ prolapse; urinary incontinence; stress; urinary bladder; overactive; surgical mesh

\section{Introduction}

Pelvic organ prolapse (POP) includes prolapse of the anterior, apical, and posterior compartments. Anterior vaginal wall is the vaginal site most commonly affected by prolapse. Instead of an isolated defect, anterior vaginal wall prolapse is highly associated with apical prolapse [1,2]. Concomitant apical suspension during anterior vaginal wall repair was demonstrated to reduce the recurrence rate of pelvic organ prolapse (POP) [3].

Higher burden of climacteric symptoms is associated with POP compared without POP [4]. Thus, the clinical outcome of POP reconstruction is an important issue in postmenopausal women. POP reconstruction can be performed by transvaginal and transabdominal approaches. Transvaginal mesh reconstruction for anterior/apical POP includes transobturator and sacrospinous mesh fixations, such as the Perigee (American Medical 
Systems, Minnetonka, MN, USA) and Uphold (Boston Scientific, Natick, MA, USA) systems [5-7]. Despite the above systems are not available in the market owing to the FDA warning [8]. However, some self-tailored vaginal mesh [9-12] and transvaginal mesh kits, such as Calistar system (Promedon, Argentine [13,14], Surelift system (Neomedic International, Terrassa, Spain [15]), Pelvimesh system (Herniamehs, Italy [16]), and Seratom ${ }^{\circledR}$ PA system (Seratex ${ }^{\circledR}$ PA B2 type, Serag-Wiessner KG, Naila, Germany [17]) are still in use. In the era of native tissue repair, autologous rectus fascia or fascia lata are used for transobturator or sacrospinous fixation [18-21]. Thus, the data of predictors of clinical outcome after transvaginal mesh reconstruction should be important for preoperative consultation.

To our knowledge, there was only one study mentioned about the comparison of POP surgery between transobturator and sacrospinous mesh fixation, and the predictor of clinical outcome was not analyzed in the study [22]. Therefore, the primary objective of this study was to identify predictors for clinical outcome in women who underwent POP reconstruction, especially with transobturator or sacrospinous mesh fixation.

\section{Methods}

Medical records of all consecutive women with Pelvic Organ Prolapse Quantification stage II or higher anterior/apical compartment prolapse, who were admitted to the department of Obstetrics and Gynecology of a tertiary referral center for POP reconstruction were reviewed. Those patients who did not undergo transobturator (i.e., the Perigee system) or sacrospinous (i.e., the Uphold system) mesh fixation were excluded in this study. In general, uncontrolled diabetes is a contraindication of vaginal mesh surgery in our hospital. The research ethics review committee of this hospital approved this study.

Transobturator mesh fixation was available between January 2011 and October 2016; however, sacrospinous mesh fixation was available between June 2015 and May 2019 in the hospital. That is, patients received transobturator mesh fixation between January 2011 and June 2015, and patients received sacrospinous mesh fixation between October 2016 and May 2019. Between June 2015 and October 2016, the choice of sacrospinous or transobturator mesh fixation was made at each surgeon's discretion.

\subsection{Operative Technique}

\subsubsection{Transobturator Mesh Fixation}

After hydrodissection, a vertical midline incision was made on the anterior vaginal wall. The vaginal epithelium and the full-thickness muscularis layer were dissected from the bladder wall. The vesicovaginal space was opened bilaterally until the plane near the ischial spine. Frequently, the anterior wall prolapse was plicated with absorbable sutures to reduce the area of the cystocele. Four cutaneous incisions were made; 2 superior incisions were made at the level of the clitoris at the upper medial edge of the obturator foramen and 2 inferior incisions were made $3 \mathrm{~cm}$ inferior and $2 \mathrm{~cm}$ lateral to the superior incisions. Superior trocars were inserted through the incision wound, penetrating the subcutaneous tissue, passing through the obturator membrane, and emerging from the vaginal incision wound with finger guidance. With the similar method, the inferior mesh arms were attached to the pelvic side wall at the level of the arcus tendineus fasciae pelvis near the ischial spine. The central part of the mesh was placed under the bladder, laid flat on the anterior vagina wall, and fixed loosely. The vaginal incision wound was closed with two layers of delayed absorbable suture [7].

\subsubsection{Sacrospinous Mesh Fixation}

After hydrodissection, a longitudinal midline vaginal incision was made with blunt dissection of the vaginal mucosa from its underlying fascia until identifying the sacrospinous ligament. With the aid of the Capio suture capturing device (Boston Scientific, Natick, MA, USA), the mesh arms were introduced and fixed at the bilateral sacrospinous ligaments. Instead of direct visualization, the location of mesh fixation was identified by palpation (about two finger breaths medial to the ischial spine). The central part of the mesh was 
sutured to the bladder wall and paracervical ring or vaginal vault. After adjusting the tension of the mesh, the incision wound was closed with two layers of delayed absorbable sutures [23].

Medical records, including obstetric and gynecologic history, body mass index, systemic disease, previous urogynecologic surgery history, coexistent overactive bladder syndrome $(\mathrm{OAB}), 20 \mathrm{~min}$ pad test, and urodynamic studies, were reviewed. In general, patients were requested to visit the outpatient clinic 7 days, 14 days, 1 month, and 3 months after surgery, and then 6-monthly thereafter. Stress urinary incontinence (SUI) was defined as the complaint of involuntary loss of urine on effort, physical exertion, sneezing, or coughing [24]. Postoperative OAB was defined if the patient received antimuscarinics or beta-3 agonist for OAB treatment during follow-up. The date of first dose of antimuscarinics or beta- 3 agonist was defined as the onset date of postoperative OAB. The date of SUI-free or OAB-free interval was calculated from the date of surgery to the date of documented SUI, OAB or the date of last follow-up. Detrusor overactivity was defined if evidence of involuntary detrusor contractions during filling cystometry [24]. Voiding dysfunction (VD) was defined as the following symptoms during or following the act of micturition, including hesitancy, slow steam, intermittency, straining to void, spraying of urinary stream, feeling of incomplete emptying, need to immediately revoid, postmicturition leakage, position-dependent micturition, dysuria or urinary retention [24].

Multichannel urodynamic equipment (Life-Tech, Houston, TX, USA) with computer analysis and Urovision (Urolab Janus System V, Houston) was used for women with coexistent lower urinary tract symptoms or excluding occult urodynamic stress incontinence. All terminology conformed to the standards recommended by the International Continence Society and Urodynamic Society [24]. All data were interpreted by a single observer to avoid interobserver variability.

The Stata software program (Version 11.0; Stata Corp, College Station, TX, USA) was used for statistical analyses. Chi-square test, Fisher's exact test, Wilcoxon rank-sum test, or McNemar's test were employed for statistical analysis. The survival curve was estimated using the Kaplan-Meier method. Multivariable Cox proportional hazards model was performed by using all variables with $p<0.10$ in the univariate analysis [25]. A $p$ value of less than 0.05 was considered statistically significant. The receiver operating characteristic curve (ROC) analysis was performed to identify the optimal cut-off value for differentiation.

\section{Results}

Between January 2011 and May 2019, a total of 206 consecutive women were reviewed, including 68 women who received POP reconstruction with transobturator mesh fixation and 138 women who underwent POP reconstruction with sacrospinous mesh fixation. The baseline characteristics of the patients are listed in Table 1. The median postoperative follow-up interval of the transobturator group (6.8 months, 25-75 interquartile range $=2.4$ to 30.8 months) was longer than the sacrospinous group (4.7 months, 25-75 interquartile range $=2.5$ to 11.6 months, $p=0.01)$. A total of $161(78.2 \%)$ patients underwent preoperative urodynamic studies. Most patients were menopausal. Except $\geq$ stage II uterine prolapse and rectocele rates, detrusor pressure at maximum flow rate, vaginal total hysterectomy, posterior colporrhaphy and follow-up interval, there were no between-group differences in the other baseline characteristics (Table 1). There was no significant between-group difference in operative time and intraoperative blood loss (Table 1).

Five surgeons were involved in this study (Table 1). Between June 2015 and October 2016, fifty-seven patients underwent sacrospinous or transobturator mesh fixation according to each surgeon's discretion. However, two surgeons preferred to perform transobturator mesh fixation (transobturator/sacrospinous case ratio $=11 / 4$ ), and three surgeons tended to perform sacrospinous mesh fixation (transobturator/sacrospinous case ratio $=8 / 34)(p<0.001)$. In addition, the presence of apical prolapse was a negative predictor of the use of transobturator mesh fixation (odds ratio $=0.26,95 \%$ confidence 
interval $(\mathrm{CI})=0.09$ to $0.76, p=0.01$ ). That is, those patients without concomitant apical prolapse tended to undergo transobturator mesh fixation.

Table 1. Baseline and peri-operative data of women who underwent surgery with transobturator or sacrospinous mesh fixation $(n=206)$.

\begin{tabular}{|c|c|c|c|}
\hline Variables & Transobturator $(n=68)$ & Sacrospinous $(n=138)$ & $+p$ \\
\hline Age (years) & $64.1 \pm 8.0$ & $64.4 \pm 9.9$ & 0.56 \\
\hline Menopause & $65(96)$ & $120(87)$ & 0.08 \\
\hline $\operatorname{BMI}\left(\mathrm{kg} / \mathrm{m}^{2}\right)$ & $25.0 \pm 3.0$ & $24.8 \pm 3.7$ & 0.50 \\
\hline Parity & $3.1 \pm 1.2$ & $3.1 \pm 1.2$ & 0.93 \\
\hline Diabetes & $15(22)$ & $42(30)$ & 0.21 \\
\hline Hypertension & $41(60)$ & $64(46)$ & 0.06 \\
\hline Prior hysterectomy & $10(15)$ & $14(10)$ & 0.34 \\
\hline Prior POP surgery & $6(9)$ & $10(7)$ & 1.00 \\
\hline$\geq$ stage II cystocele & $67(99)$ & $131(95)$ & 0.67 \\
\hline$\geq$ stage II uterine prolapse & $47(69)$ & $126(91)$ & 0.005 \\
\hline$\geq$ stage II rectocele & $30(44)$ & $88(65)$ & 0.005 \\
\hline Pad weight (g) & $17.5 \pm 39.5$ & $24.8 \pm 52.9$ & 0.48 \\
\hline $\mathrm{Qmax}(\mathrm{mL} / \mathrm{s})$ & $21.9 \pm 10.9$ & $19.8 \pm 11.5$ & 0.26 \\
\hline Voided volume (mL) & $292 \pm 164$ & $261 \pm 156$ & 0.25 \\
\hline Post-void residual (mL) & $121 \pm 84$ & $113 \pm 81$ & 0.62 \\
\hline PdetQmax $\left(\mathrm{cmH}_{2} \mathrm{O}\right)$ & $27.0 \pm 17.1$ & $35.1 \pm 19.2$ & 0.01 \\
\hline Detrusor overactivity & $14(21)$ & $18(13)$ & 0.052 \\
\hline $\operatorname{MUCP}\left(\mathrm{cmH}_{2} \mathrm{O}\right)$ & $60.1 \pm 32.0$ & $72.3 \pm 44.8$ & 0.10 \\
\hline SUI & $28(37)$ & $62(45)$ & 0.76 \\
\hline OAB & $13(19)$ & $35(24)$ & 0.64 \\
\hline VD & $15(22)$ & $45(33)$ & 0.12 \\
\hline VTH & $29(43)$ & $39(28)$ & $<0.001$ \\
\hline MUS & $7(10)$ & $20(14)$ & 0.40 \\
\hline Posterior colporrhaphy & $37(54)$ & $109(79)$ & $<0.001$ \\
\hline Operative time (mins) & $108 \pm 39$ & $119 \pm 36$ & 0.65 \\
\hline Blood loss $(\mathrm{mL})$ & $101 \pm 104$ & $142 \pm 211$ & 0.17 \\
\hline \multicolumn{4}{|l|}{ Perioperative Complications } \\
\hline Bladder perforation & $0(0)$ & $1(1)$ & 1.00 \\
\hline Massive bleeding & $0(0)$ & $1(1)$ & 1.00 \\
\hline \multicolumn{4}{|l|}{ Clavien-Dindo classification } \\
\hline Grade II & $0(0)$ & $1(1)$ & 1.00 \\
\hline Grade IIIb & $0(0)$ & $1(1)$ & 1.00 \\
\hline Follow-up interval (months) & $19.1 \pm 23.3$ & $8.2 \pm 8.7$ & 0.01 \\
\hline Recurrence of POP & $4(6)$ & $8(2)$ & $0.42 \ddagger$ \\
\hline Mesh extrusion & $5(7)$ & $8(9)$ & $0.97 \ddagger$ \\
\hline Dysuria/UTI & $7(10)$ & $9(2)$ & 0.34 \\
\hline De novo dyspareunia & $1(1)$ & $1(1)$ & 0.55 \\
\hline \multicolumn{4}{|l|}{ Surgeon } \\
\hline A & $19(28)$ & $86(62)$ & $<0.001$ \\
\hline B & $33(49)$ & $26(19)$ & \\
\hline $\mathrm{C}$ & $7(10)$ & $23(17)$ & \\
\hline $\mathrm{D}$ & $9(13)$ & $0(0)$ & \\
\hline $\mathrm{E}$ & $0(0)$ & $3(2)$ & \\
\hline $\begin{array}{l}\text { Values are expressed as mean } \pm \text { stan } \\
\text { imum urethral closure pressure. ML } \\
\text { trusor pressure at maximum flow }\end{array}$ & $\begin{array}{l}\mathrm{rd} \text { deviation or number (pe } \\
=\text { mid-urethral sling. OAB }\end{array}$ & $\begin{array}{l}\text { tage). } \mathrm{BMI}=\text { body mass ind } \\
\text { veractive bladder syndrome } \\
\text { e. } Q \mathrm{max}=\text { maximum flow }\end{array}$ & $\begin{array}{l}\text { MUCP }= \\
\text { etQmax }\end{array}$ \\
\hline
\end{tabular}

Probabilities of POP recurrence (Figure 1A), mesh extrusion (Figure 1B), postoperative SUI (Figure 1C), and postoperative OAB (Figure 1D) did not differ between the sacrospinous and transobturator groups (log-rank test, $p=0.42,0.97,0.24$ and 0.75 , respectively). 


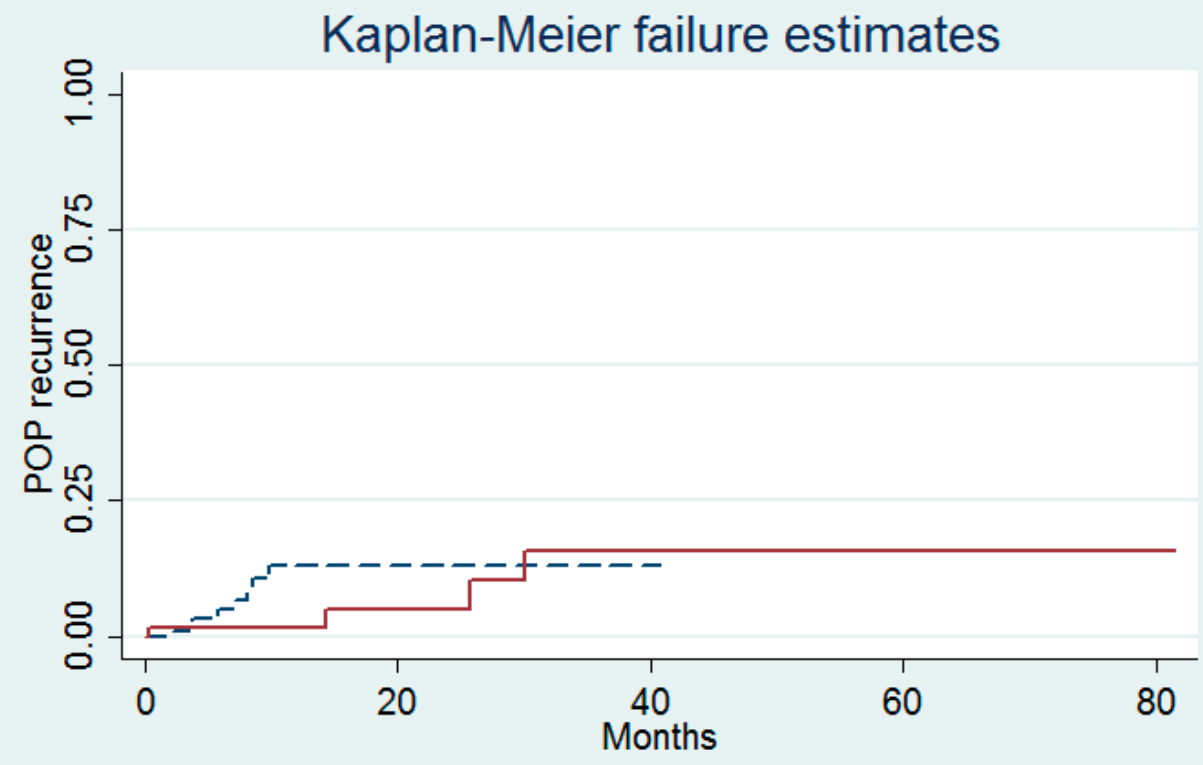

Number at risk

\begin{tabular}{|c|c|c|c|c|c|c|c|c|}
\hline \multicolumn{2}{|c|}{$\begin{array}{lc}\text { Sacrospinous } & 138 \\
\text { Transobturator } & 68\end{array}$} & $\begin{array}{l}(8) \\
(2)\end{array}$ & $\begin{array}{l}15 \\
21\end{array}$ & $\begin{array}{l}(0) \\
(2)\end{array}$ & $\begin{array}{l}1 \\
9\end{array}$ & $\begin{array}{l}(0) \\
\text { (0) }\end{array}$ & $\begin{array}{l}0 \\
3\end{array}$ & $\begin{array}{l}(0) \\
(0)\end{array}$ \\
\hline
\end{tabular}

(A)

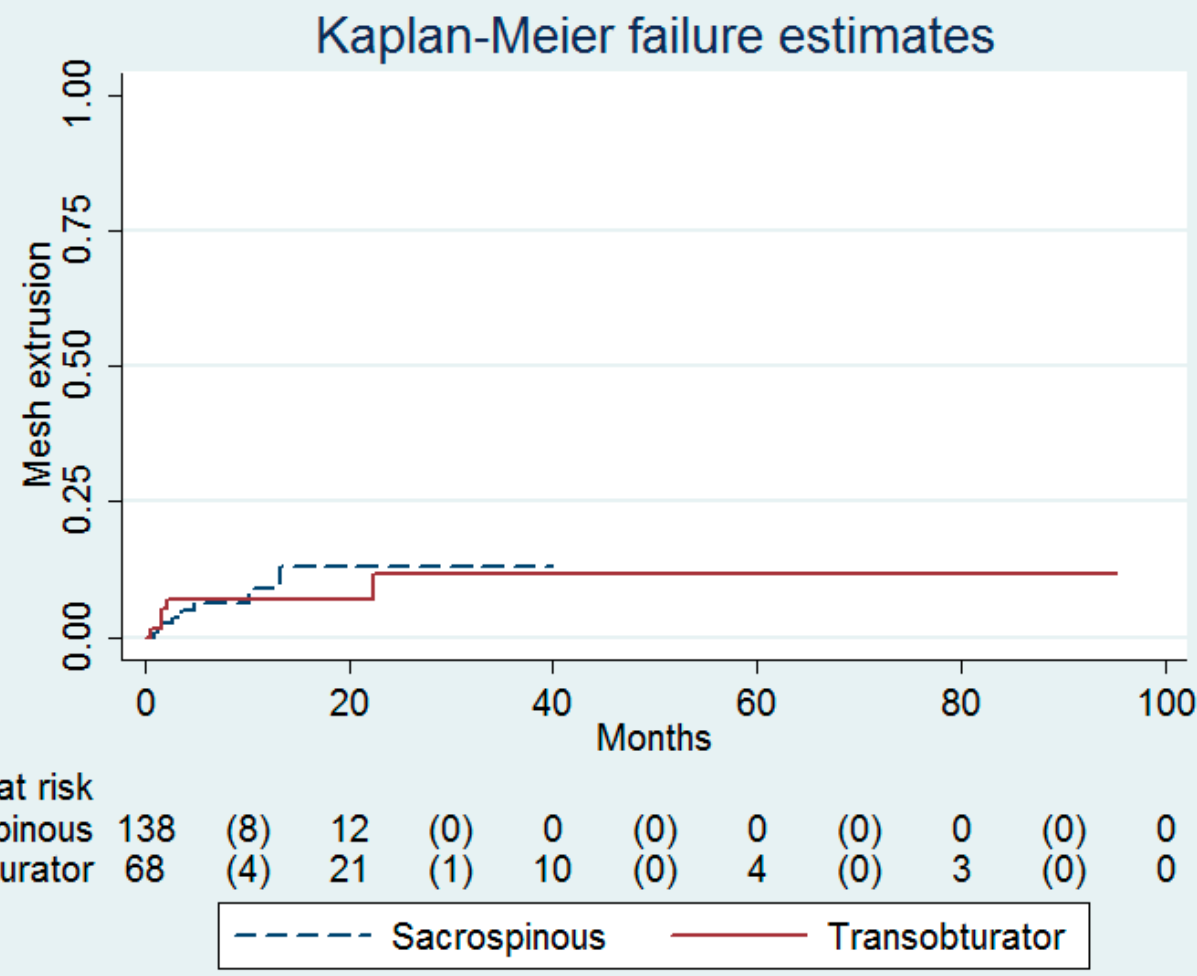

(B)

Figure 1. Cont. 

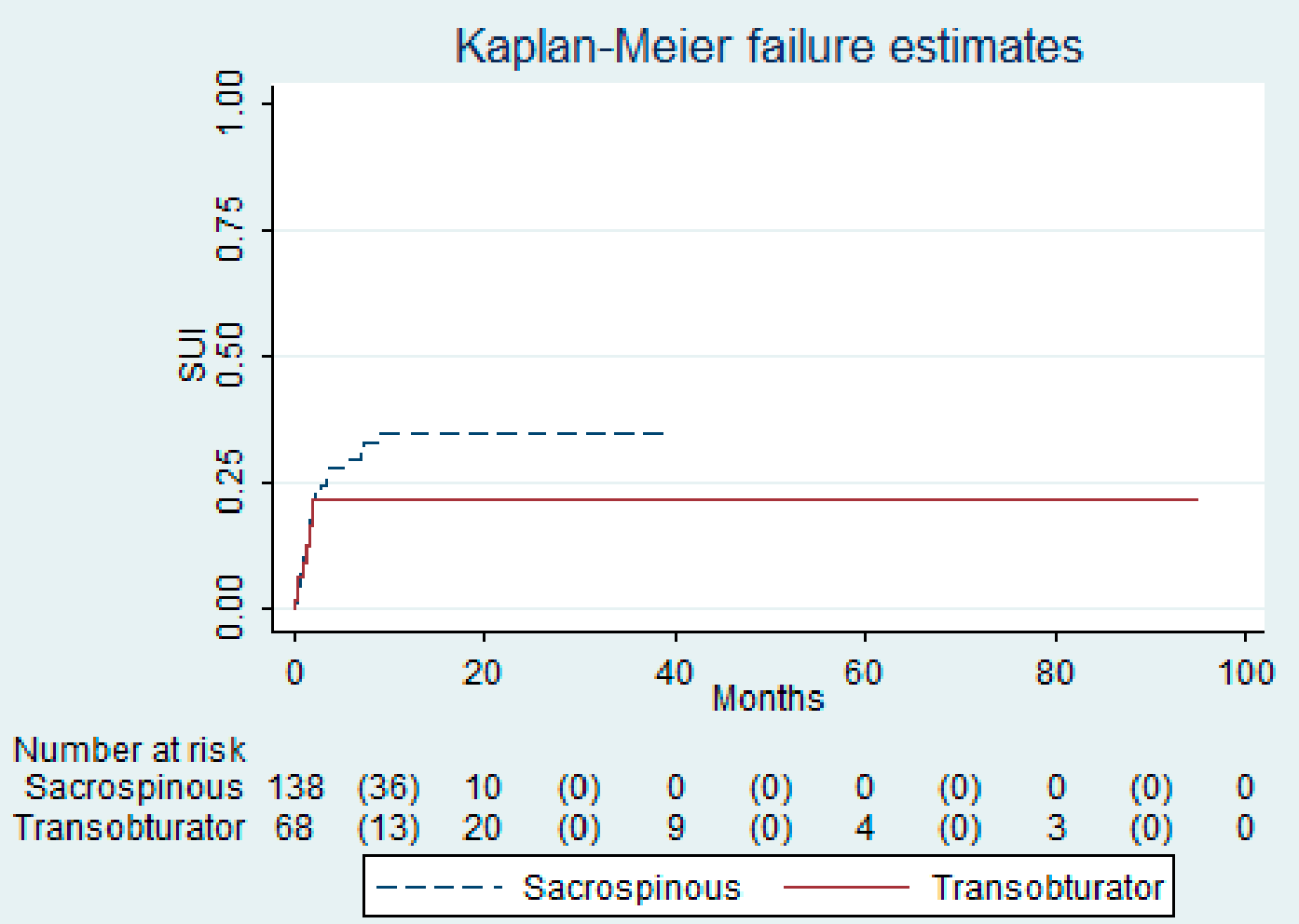

(C)

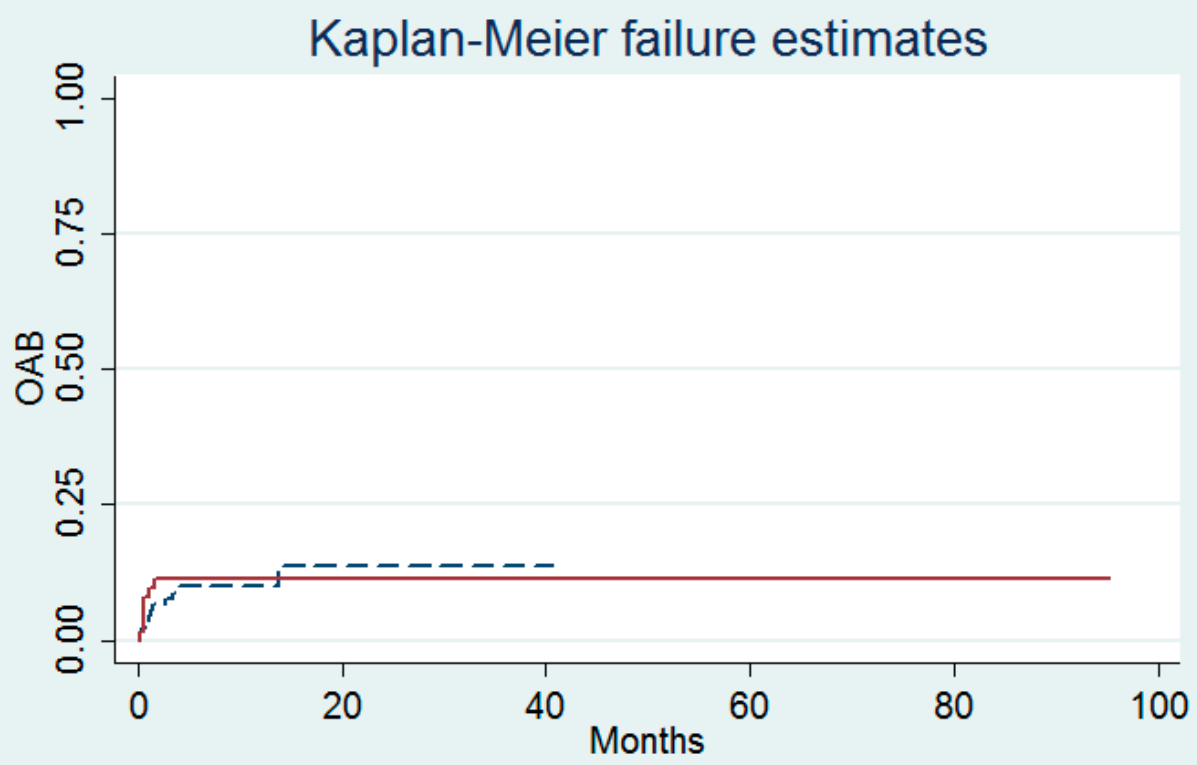

Number at risk

$\begin{array}{lllllllllll}\text { Sacrospinous } 138 & (12) & 15 & (0) & 1 & (0) & 0 & (0) & 0 & (0) & 0\end{array}$

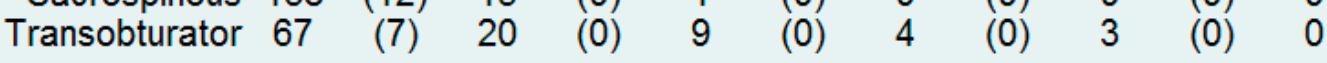

- - - - Sacrospinous Transobturator

(D)

Figure 1. Probabilities of (A) recurrence of pelvic organ prolapse (POP), (B) mesh extrusion, (C) stress urinary incontinence (SUI) and (D) overactive bladder syndrome (OAB) after the transobturator $(n=68)$ or sacrospinous $(n=138)$ mesh fixations. 
Multivariable Cox proportional hazards model revealed that the stage of cystocele (hazard ratio $=4.56$ ) and surgeon $\mathrm{E}$ (hazard ratio $=804.6$ ) were the independent predictors of POP recurrence (Table 2). Stage of cystocele $\geq 4$ was determined as an optimum cut-off value for predicting POP recurrence, which had an area under the ROC curve of 0.74 (95\% $C I=0.56$ to 0.93 ; sensitivity $=58.3 \%$, specificity $=92.5 \%$, Figure $2 \mathrm{~A}$ ).

Table 2. Factors predicting recurrence of pelvic organ prolapse and mesh extrusion $(n=206)$.

\begin{tabular}{|c|c|c|c|c|c|c|c|c|}
\hline \multirow[b]{3}{*}{ Variables } & \multicolumn{4}{|c|}{ Recurrence of POP } & \multicolumn{4}{|c|}{ Mesh Extrusion } \\
\hline & \multirow{2}{*}{$\begin{array}{c}\text { Univariate } \\
\text { HR }(95 \% \text { CI) }\end{array}$} & \multicolumn{3}{|c|}{ Multivariable } & \multirow{2}{*}{$\begin{array}{c}\text { Univariate } \\
\text { HR }(95 \% \text { CI) }\end{array}$} & \multicolumn{3}{|c|}{ Multivariable } \\
\hline & & $p+$ & HR $(95 \%$ CI $)$ & $p \ddagger$ & & $p+$ & HR $(95 \%$ CI $)$ & $p \ddagger$ \\
\hline $\begin{array}{l}\text { Transobturator } \\
\text { method }\end{array}$ & $0.60(0.17,2.11)$ & 0.43 & - & - & $0.98(0.31,3.08)$ & 0.97 & - & - \\
\hline Age (years) & $1.03(0.97,1.09)$ & 0.40 & - & - & $0.94(0.89,1.00)$ & 0.04 & $0.94(0.89,1.00)$ & 0.04 \\
\hline Menopause & $6.94 \times 10^{15}(0$, infinity $)$ & 1.00 & - & - & $1.17(0.15,9.09)$ & 0.88 & - & - \\
\hline Parity & $1.17(0.76,1.80)$ & 0.48 & - & - & $1.04(0.68,1.61)$ & 0.84 & - & - \\
\hline BMI $\left(\mathrm{kg} / \mathrm{m}^{2}\right)$ & $1.07(0.92,1.24)$ & 0.39 & - & - & $0.98(0.84,1.14)$ & 0.80 & - & - \\
\hline Hypertension & $1.41(0.45,4.46)$ & 0.56 & - & - & $1.64(0.53,5.01)$ & 0.39 & - & - \\
\hline Diabetes & $1.04(0.28,3.86)$ & 0.95 & - & - & $1.31(0.40,4.26)$ & 0.66 & - & - \\
\hline $\begin{array}{c}\text { Prior } \\
\text { hysterectomy }\end{array}$ & $0.57(0.07,4.46)$ & 0.59 & - & - & $0.61(0.08,4.72)$ & 0.64 & - & - \\
\hline $\begin{array}{c}\text { Prior POP } \\
\text { surgery }\end{array}$ & $1.21(0.16,9.43)$ & 0.85 & - & - & $1.10(0.14,8.46)$ & 0.93 & - & - \\
\hline Cystocele stage & $6.17(2.25,16.91)$ & $<0.001$ & $8.80(2.15,36.09)$ & 0.003 & $1.01(0.44,2.29)$ & 0.99 & - & - \\
\hline $\begin{array}{l}\text { Apical prolapse } \\
\text { stage } \S\end{array}$ & $2.78(1.44,5.33)$ & 0.002 & (1) & - & $0.74(0.46,1.19)$ & 0.22 & - & - \\
\hline Pad weight (g) & $1.00(0.99,1.02)$ & 0.45 & - & - & $1.00(0.99,1.01)$ & 0.90 & - & - \\
\hline $\mathrm{Qmax}(\mathrm{mL} / \mathrm{s})$ & $1.03(0.98,1.08)$ & 0.29 & - & - & $0.99(0.94,1.04)$ & 0.71 & - & - \\
\hline $\begin{array}{l}\text { Voided volume } \\
\qquad(\mathrm{mL})\end{array}$ & $1.00(1.00,1.00)$ & 0.99 & - & - & $1.00(1.00,1.00)$ & 0.30 & - & - \\
\hline $\begin{array}{c}\text { Post-void } \\
\text { residual }(\mathrm{mL})\end{array}$ & $1.01(1.00,1.01)$ & 0.16 & - & - & $1.01(1.00,1.01)$ & 0.11 & - & - \\
\hline $\begin{array}{l}\text { PdetQmax } \\
\left(\mathrm{cmH}_{2} \mathrm{O}\right)\end{array}$ & $0.99(0.95,1.05)$ & 0.81 & - & - & $0.98(0.93,1.02)$ & 0.26 & - & - \\
\hline $\begin{array}{c}\text { Detrusor } \\
\text { overactivity }\end{array}$ & $0.93(0.20,4.36)$ & 0.92 & - & - & $0.89(0.19,4.14)$ & 0.89 & - & - \\
\hline $\operatorname{MUCP}\left(\mathrm{cmH}_{2} \mathrm{O}\right)$ & $1.01(0.99,1.02)$ & 0.28 & - & - & $1.00(0.98,1.01)$ & 0.74 & - & - \\
\hline SUI & $0.87(0.26,2.88)$ & 0.81 & - & - & $2.03(0.68,6.04)$ & 0.21 & - & - \\
\hline $\mathrm{OAB}$ & $1.06(0.29,3.94)$ & 0.93 & - & - & $0.96(0.26,3.47)$ & 0.95 & - & - \\
\hline VD & $3.16(1.02,9.84)$ & 0.047 & $2.77(0.76,10.10)$ & 0.12 & $0.81(0.22,2.94)$ & 0.75 & - & - \\
\hline VTH & $0.24(0.03,1.86)$ & 0.17 & - & - & $0.49(0.11,2.23)$ & 0.36 & - & - \\
\hline $\begin{array}{l}\text { Mid-urethral } \\
\text { sling } \\
\text { Surgeon II }\end{array}$ & $1.31(0.29,6.03)$ & 0.73 & - & - & $0.54(0.07,4.15)$ & 0.55 & - & - \\
\hline A (reference) & 1.00 & - & 1.00 & - & 1.00 & - & - & - \\
\hline B & $9 ., 95(1.19,83.1)$ & 0.03 & $3.90(0.44,34.43)$ & 0.22 & $1.79(0.52,6.19)$ & 0.36 & - & - \\
\hline $\mathrm{C}$ & $14.1(1.57,127.08)$ & 0.02 & $2.90(0.21,39.88)$ & 0.43 & $1.90(0.45,7.98)$ & 0.38 & - & - \\
\hline $\mathrm{D}$ & $8.66 \times 10^{-19}(-,-)$ & - & $9.89 \times 10^{-19}(-,-)$ & - & $\begin{array}{c}2.01 \times 10^{-15}(0, \\
\text { infinity })\end{array}$ & 1.00 & - & - \\
\hline $\mathrm{E}$ & $36.07(2.20,591.54)$ & 0.01 & $\begin{array}{c}804.60(21.63 \\
29,924.48)\end{array}$ & $<0.001$ & $\begin{array}{c}2.00 \times 10^{-15}(0, \\
\text { infinity })\end{array}$ & 1.00 & - & - \\
\hline
\end{tabular}

$\mathrm{CI}=$ confidence interval. $\mathrm{HR}=$ hazard ratio. The other abbreviations are the same as in Table 1. + Cox proportional hazards model. $\ddagger$ Multivariable Cox proportional hazards modeling was performed using all variables with $p<$ 0.10 in the univariate analysis. § Owing to a significant correlation between cystocele stage and apical prolapse stage (Spearman's rho $=0.39, p<0.0001$ ), apical prolapse stage was excluded in the multivariable Cox proportional hazards modeling for predicting recurrence of pelvic organ prolapse. II Experienced POP surgical cases: surgeon $\mathrm{A}>$ surgeon $\mathrm{B}>$ surgeon $\mathrm{C}>$ surgeon $\mathrm{D}>$ surgeon $\mathrm{E}$. 


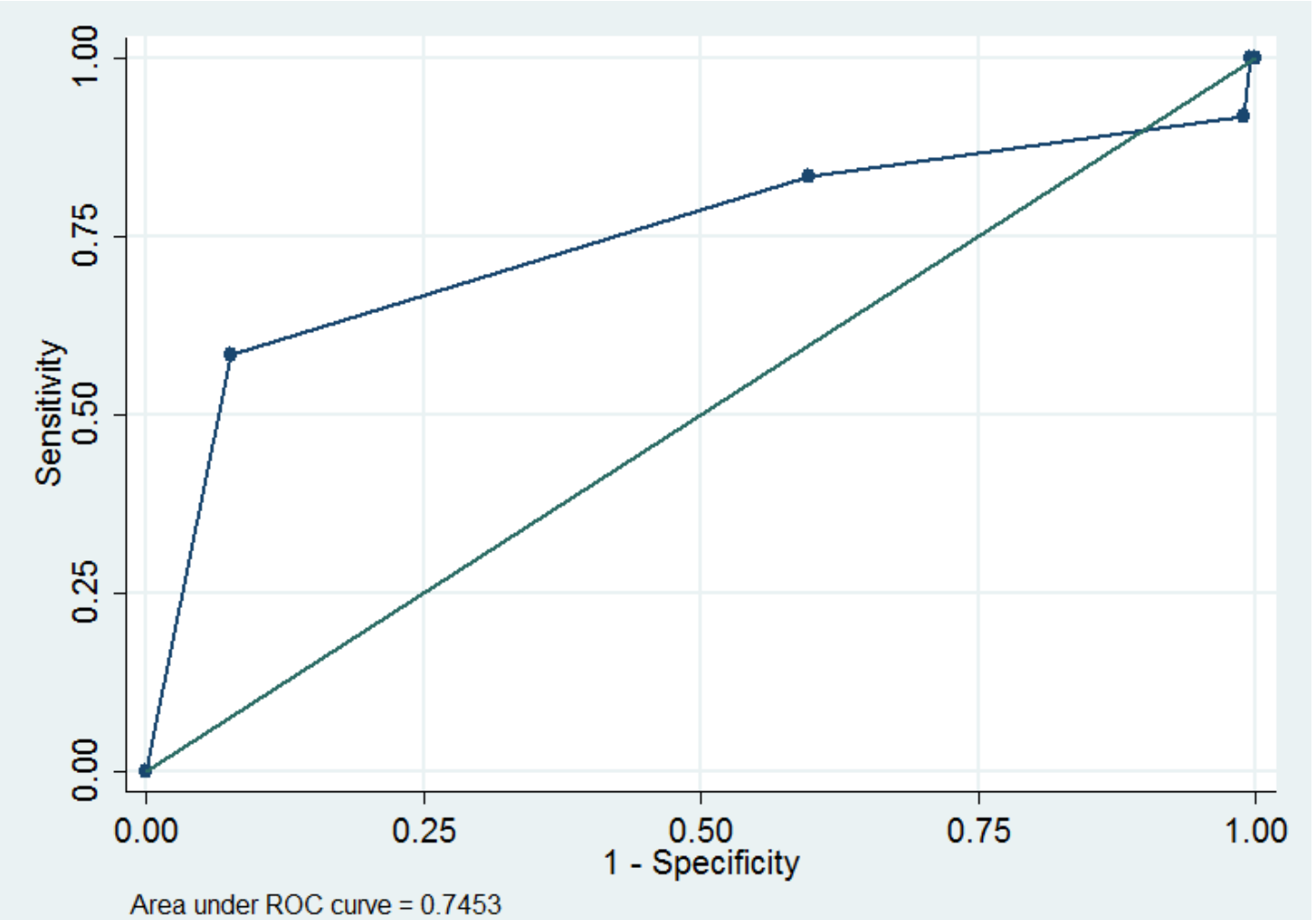

(A)

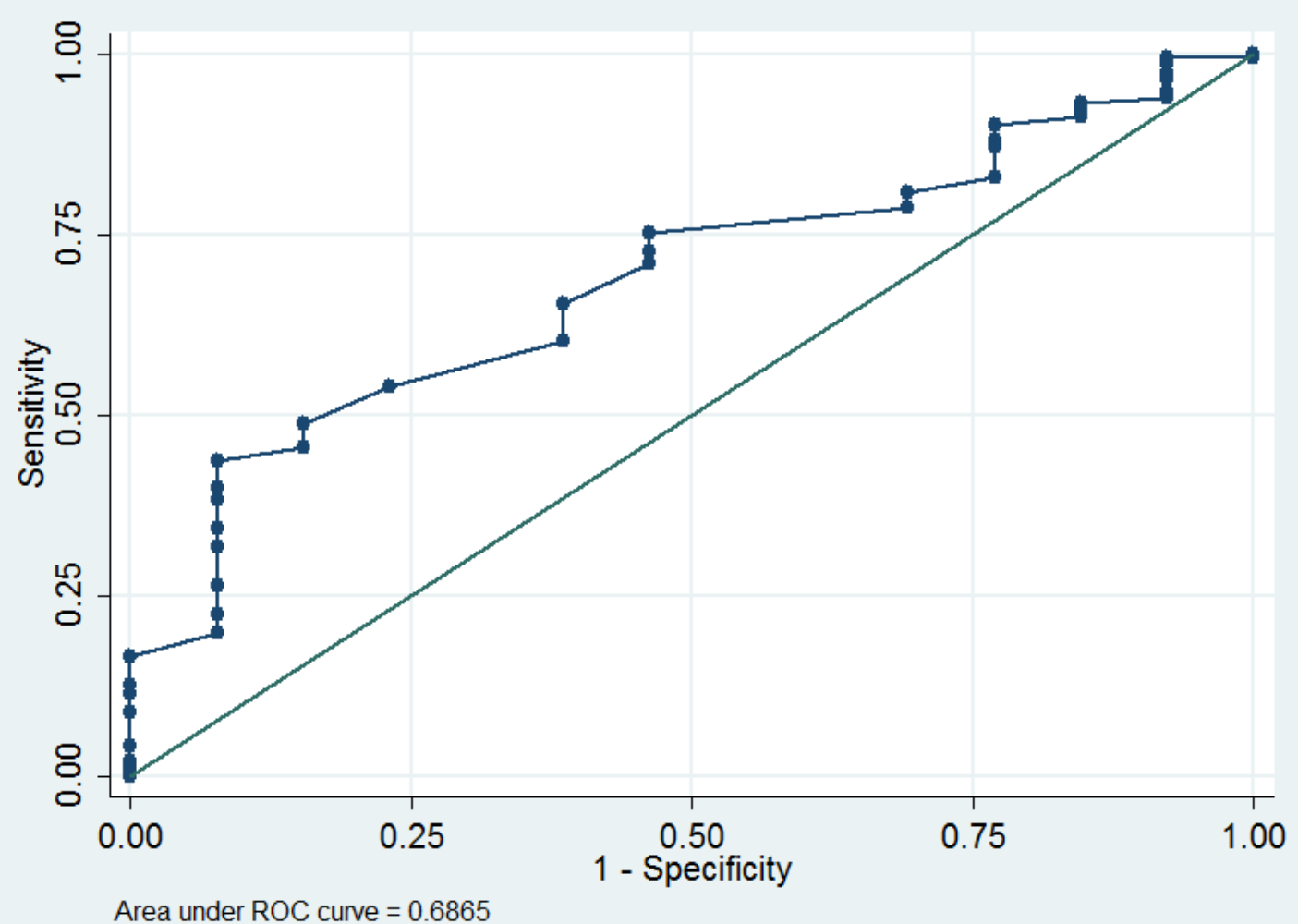

(B)

Figure 2. The receiver operating characteristic curves for (A) stage of cystocele as a predictor of recurrence of pelvic organ prolapse, and (B) age as a predictor of mesh extrusion. 
Multivariable Cox proportional hazards model revealed that age (hazard ratio $=0.94$ ) was the only predictor of mesh extrusion (Table 2). Age $\leq 67$ years was determined as an optimum cut-off value for predicting a higher rate of mesh extrusion, which had an area under the ROC curve of 0.69 (95\% CI $=0.56$ to 0.82 ; sensitivity $=43.5 \%$, specificity $=92.3 \%$, Figure 2B). If we used multivariable logistic regression analysis, the follow-up interval (odds ratio $=1.03,95 \% \mathrm{CI}=1.02$ to $1.05, p=0.02$ ) was also an independent predictor of mesh extrusion in additional to young age (odds ratio $=0.94,95 \% \mathrm{CI}=0.88$ to $1.00, p=0.04$ ).

Multivariable Cox proportional hazards model also revealed that Qmax (hazard ratio $=1.03$ ) was the sole predictor of postoperative SUI (Table 3). Qmax $\geq 19.2 \mathrm{~mL} / \mathrm{s}$ was determined as an optimum cut-off value for predicting postoperative SUI, which had an area under the ROC curve of $0.59(95 \% \mathrm{CI}=0.48$ to 0.69 ; sensitivity $=62.5 \%$, specificity $=55.1 \%$, Figure 3 ).

Table 3. Factors predicting postoperative stress urinary incontinence and overactive bladder syndrome $(n=206)$.

\begin{tabular}{|c|c|c|c|c|c|c|c|c|}
\hline \multirow[b]{3}{*}{ Variables } & \multicolumn{4}{|c|}{ Postoperative SUI } & \multicolumn{4}{|c|}{ Postoperative OAB } \\
\hline & \multirow{2}{*}{$\begin{array}{c}\text { Univariate } \\
\text { HR }(95 \% \text { CI) }\end{array}$} & \multicolumn{3}{|c|}{ Multivariable } & \multirow{2}{*}{$\begin{array}{c}\text { Univariate } \\
\text { HR (95\% CI) }\end{array}$} & \multicolumn{3}{|c|}{ Multivariable } \\
\hline & & $p+$ & HR $(95 \%$ CI) & $p \ddagger$ & & $p+$ & HR (95\% CI) & $p \ddagger$ \\
\hline $\begin{array}{l}\text { Transobturator } \\
\text { fixation }\end{array}$ & $0.69(0.36,1.29)$ & 0.24 & - & - & $1.17(0.46,2.98)$ & 0.75 & - & - \\
\hline Age (years) & $1.01(0.98,1.04)$ & 0.74 & - & - & $1.02(0.97,1.07)$ & 0.47 & - & - \\
\hline Menopause & $0.91(0.36,2.29)$ & 0.84 & - & - & $1.95(0.26,14.63)$ & 0.52 & - & - \\
\hline Parity & $0.92(0.72,1.17)$ & 0.48 & - & - & $0.91(0.63,1.33)$ & 0.64 & - & - \\
\hline BMI $\left(\mathrm{kg} / \mathrm{m}^{2}\right)$ & $1.04(0.96,1.12)$ & 0.33 & - & - & $0.93(0.81,1.07)$ & 0.31 & - & - \\
\hline Hypertension & $0.98(0.56,1.72)$ & 0.96 & - & - & $0.57(0.23,1.45)$ & 0.24 & - & - \\
\hline Diabetes & $1.40(0.77,2.55)$ & 0.27 & - & - & $0.49(0.14,1.69)$ & 0.26 & - & - \\
\hline $\begin{array}{c}\text { Prior } \\
\text { hysterectomy }\end{array}$ & $1.48(0.66,3.28)$ & 0.34 & - & - & $3.30(1.18,9.19)$ & 0.02 & $2.58(0.91,7.28)$ & 0.07 \\
\hline $\begin{array}{c}\text { Prior POP } \\
\text { surgery }\end{array}$ & $0.80(0.25,2.57)$ & 0.71 & - & - & $1.56(0.36,6.74)$ & 0.56 & - & - \\
\hline Cystocele stage & $1.09(0.73,1.61)$ & 0.68 & - & - & $0.86(0.45,1.64)$ & 0.64 & - & - \\
\hline $\begin{array}{l}\text { Apical prolapse } \\
\text { stage }\end{array}$ & $0.93(0.73,1.19)$ & 0.56 & - & - & $0.84(0.57,1.24)$ & 0.38 & - & - \\
\hline Pad weight (g) & $0.99(0.98,1.00)$ & 0.12 & - & - & $1.00(0.99,1.01)$ & 0.45 & - & - \\
\hline Qmax (mL/s) & $1.02(1.00,1.05)$ & 0.07 & $1.03(1.00,1.07)$ & 0.04 & $1.01(0.97,1.06)$ & 0.59 & - & - \\
\hline $\begin{array}{l}\text { Voided volume } \\
\qquad(\mathrm{mL})\end{array}$ & $1.00(1.00,1.00)$ & 0.48 & - & - & $1.00(1.00,1.00)$ & 0.46 & - & - \\
\hline $\begin{array}{c}\text { Post-void } \\
\text { residual }(\mathrm{mL})\end{array}$ & $1.00(1.00,1.00)$ & 0.74 & - & - & $1.00(0.99,1.00)$ & 0.54 & - & - \\
\hline $\begin{array}{c}\text { PdetQmax } \\
\left(\mathrm{cmH}_{2} \mathrm{O}\right)\end{array}$ & $0.97(0.95,1.00)$ & 0.02 & $0.98(0.96,1.01)$ & 0.15 & $0.99(0.96,1.02)$ & 0.53 & - & - \\
\hline $\begin{array}{c}\text { Detrusor } \\
\text { overactivity }\end{array}$ & $0.43(0.15,1.21)$ & 0.11 & - & - & $1.83(0.64,5.28)$ & 0.26 & - & - \\
\hline $\operatorname{MUCP}\left(\mathrm{cmH}_{2} \mathrm{O}\right)$ & $0.99(0.98,1.00)$ & 0.01 & $0.99(0.98,1.01)$ & 0.38 & $1.00(0.99,1.01)$ & 0.90 & - & - \\
\hline SUI & $1.12(0.64,2.01)$ & 0.68 & - & - & $1.63(0.66,4.01)$ & 0.29 & - & - \\
\hline $\mathrm{OAB}$ & $1.06(0.55,2.04)$ & 0.86 & - & - & $3.13(1.27,7.71)$ & 0.01 & $3.22(1.30,7.96)$ & 0.01 \\
\hline VD & $1.38(0.77,2.49)$ & 0.28 & - & - & $1.17(0.44,3.07)$ & 0.75 & - & - \\
\hline VTH & $0.59(0.28,1.25)$ & 0.17 & - & - & $0.16(0.02,1.22)$ & 0.08 & $0.19(0.02,1.46)$ & 0.11 \\
\hline $\begin{array}{l}\text { Mid-urethral } \\
\text { sling } \\
\text { Surgeon } \S\end{array}$ & $0.13(0.02,0.92)$ & 0.04 & $0.15(0.02,1.17)$ & 0.07 & $1.85(0.61,5.58)$ & 0.27 & 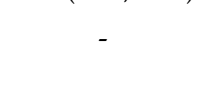 & - \\
\hline A (reference) & 1.00 & - & 1.00 & - & 1.00 & & - & - \\
\hline $\mathrm{B}$ & $0.98(0.54,1.78)$ & 0.94 & $0.99(0.44,2.02)$ & 0.98 & $0.70(0.25,1.99)$ & 0.51 & - & - \\
\hline $\mathrm{C}$ & $0.31(0.09,1.02)$ & 0.054 & $0.23(0.03,1.79)$ & 0.16 & $0.56(0.12,2.50)$ & 0.45 & - & - \\
\hline $\mathrm{D}$ & $\begin{array}{c}6.28 \times 10^{-17} \\
(0, \text { infinity })\end{array}$ & 1.00 & 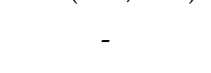 & - & $\begin{array}{c}1.69 \times 10^{-16} \\
(0, \text { infinity })\end{array}$ & 1.00 & - & - \\
\hline $\mathrm{E}$ & $\begin{array}{l}6.27 \times 10^{-17} \\
(0, \text { infinity })\end{array}$ & 1.00 & $\begin{array}{c}8.30 \times 10^{-19}(0, \\
\text { infinity })\end{array}$ & 1.00 & $\begin{array}{l}1.70 \times 10^{-16} \\
(0, \text { infinity })\end{array}$ & 1.00 & - & - \\
\hline
\end{tabular}

$\mathrm{CI}=$ confidence interval. $\mathrm{HR}$ = hazard ratio. The other abbreviations are the same as in Table 1. + Cox proportional hazards model. $\ddagger$ Multivariable backward stepwise Cox proportional hazards modeling was performed using all variables with $p<0.10$ in the univariate analysis. § Experienced POP surgical cases: surgeon A surgeon B > surgeon $\mathrm{C}>$ surgeon $\mathrm{D}>$ surgeon $\mathrm{E}$. 


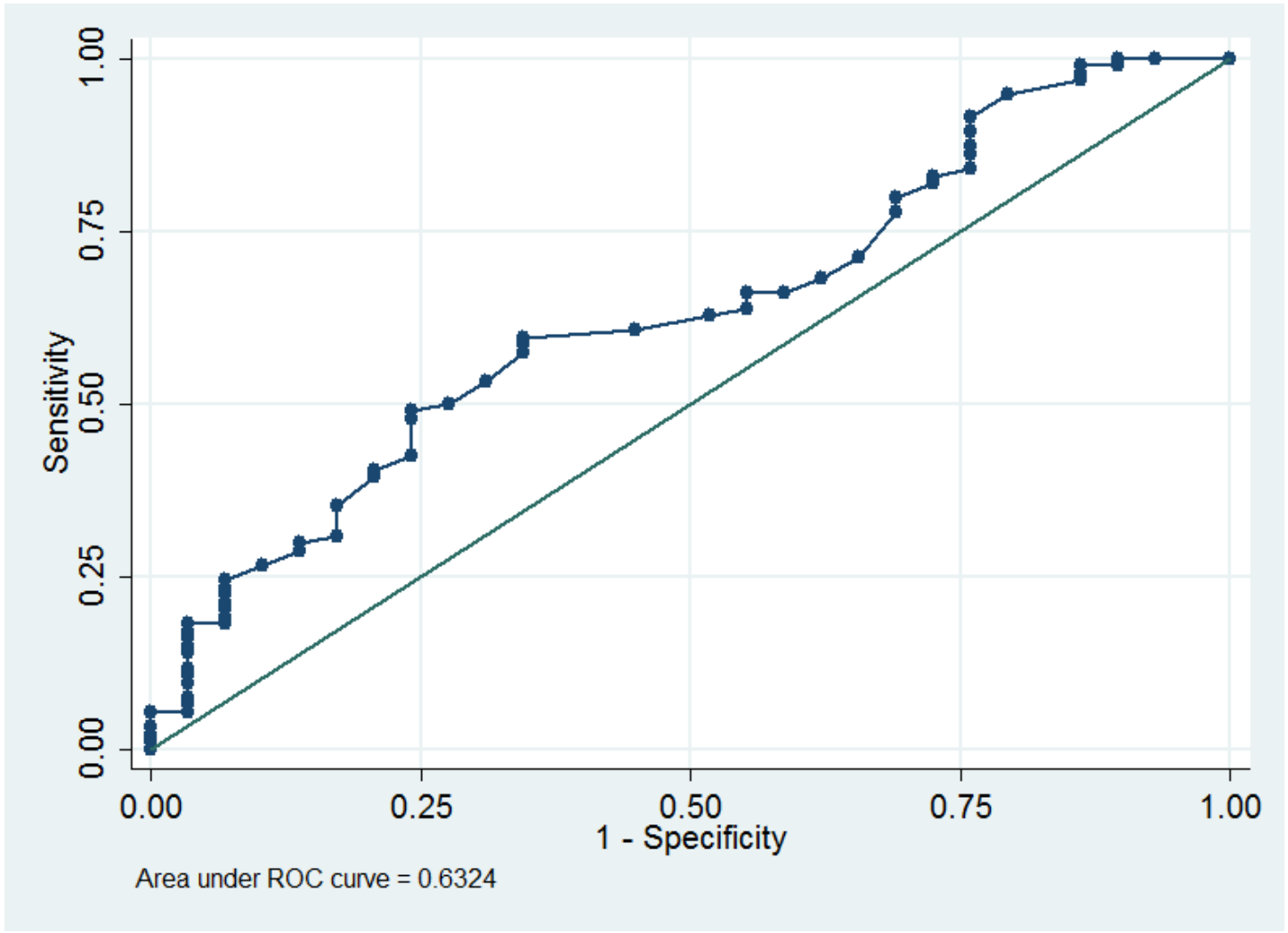

Figure 3. The receiver operating characteristic curve for the maximum flow rate as a predictor of postoperative stress urinary incontinence.

Multivariable Cox proportional hazards model revealed that preoperative OAB (hazard ratio $=3.22$ ) was the sole predictor for postoperative OAB (Table 3).

Univariate logistic regression analysis did not reveal any predictors for postoperative VD (Table 4).

Table 4. Factors predicting postoperative voiding dysfunction $(n=206)$.

\begin{tabular}{ccc}
\hline & & Univariate \\
Variables $\ddagger$ & Odds Ratio $(\mathbf{9 5} \% \mathbf{C I})$ & $p+$ \\
\hline Transobturator fixation & $0.28(0.03,2.32)$ & 0.24 \\
Age (years) & $1.02(0.95,1.11)$ & 0.57 \\
Parity & $1.05(0.57,1.93)$ & 0.89 \\
BMI $\left(\mathrm{kg} / \mathrm{m}^{2}\right)$ & $0.90(0.72,1.13)$ & 0.35 \\
Hypertension & $0.96(0.23,3.95)$ & 0.96 \\
Diabetes & $1.60(0.37,6.92)$ & 0.53 \\
Prior hysterectomy & $1.09(0.13,9.24)$ & 0.94 \\
Prior POP surgery & $1.74(0.20,15.12)$ & 0.61 \\
Cystocele stage & $0.76(0.26,2.26)$ & 0.62 \\
Apical prolapse stage & $1.50(0.72,3.13)$ & 0.27 \\
\hline Pad weight $(\mathrm{g})$ & $1.01(1.00,1.02)$ & 0.26 \\
Qmax $\left(\mathrm{mL}^{\mathrm{s}}\right)$ & $0.97(0.90,1.05)$ & 0.42 \\
Voided volume $(\mathrm{mL})$ & $1.00(0.99,1.00)$ & 0.18 \\
Post-void residual $(\mathrm{mL})$ & $1.00(0.99,1.01)$ & 0.46 \\
PdetQmax $\left(\mathrm{cmH}_{2} \mathrm{O}\right)$ & $1.02(0.98,1.06)$ & 0.39 \\
Detrusor overactivity & $1.61(0.30,8.72)$ & 0.58 \\
MUCP $\left(\mathrm{cmH}_{2} \mathrm{O}\right)$ & $1.00(0.98,1.02)$ & 0.94 \\
\hline
\end{tabular}


Table 4. Cont.

\begin{tabular}{ccc}
\hline Variables $\ddagger$ & Univariate & $p+$ \\
\hline SUI & Odds Ratio $(\mathbf{9 5 \%} \mathbf{C I})$ & 0.42 \\
OAB & $1.79(0.43,7.37)$ & 0.34 \\
VD & $2.04(0.47,8.87)$ & 0.20 \\
VTH & $2.54(0.61,10.49)$ & 0.47 \\
Mid-urethral sling & $0.46(0.06,3.83)$ & 0.32 \\
Surgeon § & $2.31(0.44,12.06)$ & - \\
A (reference) & 1.00 & 0.68 \\
B & $0.70(0.13,3.73)$ & 0.74 \\
C & $0.69(0.08,6.14)$ & - \\
D & - & - \\
E & - &
\end{tabular}

$\mathrm{CI}=$ confidence interval. The other abbreviations are the same as in Table $1 .+$ Logistic regression analysis $\ddagger$ Menopause $=1$ predicts postoperative voiding dysfunction perfectly, the variable "menopause" was omitted . $\S$ Experienced POP surgical cases: surgeon $\mathrm{A}>$ surgeon $\mathrm{B}>$ surgeon $\mathrm{C}>$ surgeon $\mathrm{D}>$ surgeon $\mathrm{E}$.

Comparison of baseline and postoperative lower urinary tract symptoms was shown in Table 5. In both groups, SUI rates did not change after surgery in women without concomitant mid-urethral sling procedures. However, OAB improved after surgery in the sacrospinous group $(p=0.0001)$. In addition, VD improved after surgery in both groups (Table 5).

Table 5. Baseline and postoperative low urinary tract symptoms $(n=206)$.

\begin{tabular}{ccccccc}
\hline & \multicolumn{2}{c}{ Transobturator $(n=68)$} & \multicolumn{3}{c}{ Sacrospinous $(\boldsymbol{n = 1 3 8 )}$} \\
Variables & Baseline & After Surgery & $\boldsymbol{p}+$ & Baseline & After Surgery & $p \dagger$ \\
\hline SUI $\ddagger$ & $20(33)$ & $13(21)$ & 0.11 & $36(31)$ & $35(30)$ & 0.89 \\
OAB & $13(19)$ & $8(12)$ & 0.20 & $35(25)$ & $12(9)$ & 0.0001 \\
VD & $15(22)$ & $1(1)$ & 0.0005 & $45(33)$ & $7(5)$ & $<0.0001$ \\
\hline
\end{tabular}

Values are expressed as numbers (percentage). The abbreviations are the same as in Table 1. † McNemar's test. $\ddagger$ Women who underwent concomitant mid-urethral slings are excluded from statistical analysis for SUI. Thus, there was 61 women in the transobturator fixation group and 118 women in the sacrospinous fixation group.

\section{Discussion}

In this study, the method of POP reconstruction (i.e., sacrospinous versus transobturator mesh fixation) is not a predictor for POP recurrence, mesh extrusion, and postoperative SUI, OAB and VD (Figure 1A-D, Tables 2-4). Similarly, Lo et al. found that sacrospinous fixation (i.e., the Elevate system, American Medical Systems, Minnetonka, MN, USA) had a similar objective and subjective cure rate, compared with transobturator fixation (i.e., the Perigee system) [22]. Kato et al. also reported that the reoperation rates due to prolapse recurrence and mesh exposure rates did not differ between the Prolift-type (i.e., anterior mesh with transobturator arms, posterior mesh with sacrospinous arms) and Uphold-type (i.e., anterior mesh with sacrospinous arms) groups [26].

Cystocele stage was a predictor for POP recurrence (hazard ratio $=4.56$, Table 3 ). Similarly, Vergeldt et al. reported that cystocele stage could predict cystocele recurrence [27]. Recently, a meta-analysis also revealed preoperative stage $2-4$ (odds ratio $=2.11, p<0.001$ ) was one significant predictor of POP recurrence [28]. We also found that stage 4 of cystocele was an optimal cut-off point for predicting POP recurrence (Figure 2A), and this finding could be used as a reference for preoperative consultation.

In our study, the least experienced surgeon (i.e., only three cases were performed by surgeon E) was a risk factor for POP recurrence (hazard ratio $=804.60, p<0.001$, Table 2). Similarly, Long et al. also reported that the POP failure rate was higher during the first 50 cases, compared with the subsequent cases (5\% vs. $1.6 \%, p=0.043)$ [29]. Price et al. reported that the majority of POP failures occur within the first three years 
following the initial operation [30]. Nonetheless, Nüssler et al. reported that the high $\mathrm{POP}$ recurrence rate was not due to insufficient experience of the surgeons performing the operation [31].

Young age was a predictor for mesh extrusion (hazard ratio $=0.94, p=0.04$, Table 2); and age $\leq 67$ years was determined as an optimum cut-off value for predicting mesh extrusion (Figure 2B). Similarly, Khrucharoen et al. reported that menopause was not a predictor for mesh extrusion (odds ratio $=1.385, p=0.15$ ) [32]. The reason for young age as a predictor for mesh extrusion was unknown. However, age-related complaints (e.g., decreased lubrication and orgasmic difficulties) could result in sexual dysfunction [33,34]. Asian middle-aged and older women tend to report less frequent sexual activity [35]. Thus, sexual dysfunction in aged women might be responsible for a lower rate of mesh extrusion. It is worth mentioning that age was not significantly associated with mesh extrusion in Ehsani et al.'s study [36]. In addition, it is worth mentioning that the follow-up time interval (odds ratio $=1.03, p=0.02$ ) was also an independent predictor of mesh extrusion. The mesh extrusion rate might increase with time.

Qmax was a predictor for postoperative SUI (hazard ratio $=1.03, p=0.04$, Table 4), especially for those women with Qmax $\geq 19.2 \mathrm{~mL} / \mathrm{s}$ (Figure 3). Similarly, Kawaguchi et al. reported that high flow rate was associated with postoperative SUI [37]. High flow rate was associated with SUI [38-40]. Therefore, it seems reasonable that Qmax is a predictor of postoperative SUI.

Preoperative $\mathrm{OAB}$ (hazard ratio $=3.22, p=0.01$ ) was an independent predictor for postoperative OAB (Table 4), which is in line with the existing studies. de Boer TA et al. also found that the absence of preoperative $\mathrm{OAB}$ was the best predictor for the absence of postoperative OAB [41,42].

OAB improved after sacrospinous mesh fixation ( $p=0.0001$, Table 5$)$. Similarly, several studies also found that an improvement of OAB after POP construction [43-45], and a reduction of $50 \%$ of urinary urgency incontinence could be expected [45]. VD improved after transobturator or sacrospinous mesh fixation (Table 5). POP surgery can improve anatomic support of the anterior compartment, thus improving VD.

Limitations of this study included a retrospective nature and limited sample size. In addition, different between-group follow-up time intervals and different surgical experience of the surgeons may bias the results. Besides, an average of two cases per month in this hospital might be not enough for surgeons to achieve surgical proficiency in POP surgery, and this can result in the bias. In general, patients with anterior compartment prolapse but without apical prolapse might be suitable to receive transobturator mesh fixation. We also found that the presence of apical prolapse was a negative predictor of the use of transobturator mesh fixation (Odds ratio $=0.26, p=0.01$ ); however, this finding might bias our results. Despite the Uphold system and the Perigee system are not available currently. However, some similar commercial kits, self-tailored meshes, and autologous fasciae remain in use for transvaginal POP reconstruction [9-21]. Thus, our results could provide as a guide for perioperative consultation, even in the era of native tissue repair.

\section{Conclusions}

Predictors of clinical outcome in women who underwent transvaginal POP reconstruction are identified. The above results can serve as a guide for preoperative consultation.

Author Contributions: Conceptualization, S.-M.H.; methodology, S.-M.H.; software, S.-M.H.; validation, T.-H.L., F.-C.T., H.-H.L. and S.-M.H.; formal analysis, S.-M.H.; investigation, S.-M.H.; resources, S.-M.H.; data curation, T.-H.L.; writing-original draft preparation, T.-H.L.; writing-review and editing, S.-M.H.; visualization, S.-M.H.; supervision, F.-C.T. and H.-H.L.; project administration, S.-M.H.; funding acquisition, S.-M.H. All authors have read and agreed to the published version of the manuscript.

Funding: This research received no external funding. 
Institutional Review Board Statement: This study was approved by the Research Ethics Review Committee of Far Eastern Memorial Hospital (No. 107159-E; approval date: 13 November 2019).

Informed Consent Statement: The Research Ethics Review Committee of Far Eastern Memorial Hospital waived the requirement for obtaining informed consent.

Data Availability Statement: The datasets of the current study are available from the corresponding author on reasonable request.

Conflicts of Interest: The authors declare no conflict of interest.

\section{References}

1. Rooney, K.; Kenton, K.; Mueller, E.R.; FitzGerald, M.P.; Brubaker, L. Advanced anterior vaginal wall prolapse is highly correlated with apical prolapse. Am. J. Obstet. Gynecol. 2006, 195, 1837-1840. [CrossRef]

2. Elliott, C.S.; Yeh, J.; Comiter, C.V.; Chen, B.; Sokol, E.R. The Predictive Value of a Cystocele for Concomitant Vaginal Apical Prolapse. J. Urol. 2013, 189, 200-203. [CrossRef]

3. Eilber, K.S.; Alperin, M.; Khan, A.; Wu, N.; Pashos, C.L.; Clemens, J.Q.; Anger, J.T. Outcomes of Vaginal Prolapse Surgery among Female Medicare Beneficiaries: The role of apical support. Obstet. Gynecol. 2013, 122, 981-987. [CrossRef]

4. Cagnacci, A.; Palma, F.; Napolitano, A.; Xholli, A. Association between pelvic organ prolapse and climacteric symptoms in postmenopausal women. Maturitas 2017, 99, 73-78. [CrossRef]

5. Rahkola-Soisalo, P.; Mikkola, T.; Altman, D.; Falconer, C.; Nordic TVM Group. Pelvic Organ Prolapse Repair Using the Uphold Vaginal Support System: 5-Year Follow-Up. Female Pelvic Med. Reconstr. Surg. 2019, 25, 200-205. [CrossRef]

6. Altman, D.; Mikkola, T.S.; Bek, K.M.; Rahkola-Soisalo, P.; Gunnarsson, J.; Engh, M.E.; Falconer, C. Pelvic organ prolapse repair using the Uphold ${ }^{\mathrm{TM}}$ Vaginal Support System: A 1-year multicenter study. Int. Urogynecol. J. 2016, 27, 1337-1345. [CrossRef]

7. $\quad$ Long, C.-Y.; Wang, C.-L.; Wu, M.-P.; Wu, C.-H.; Lin, K.-L.; Liu, C.-M.; Tsai, E.-M.; Shen, C.-J. Comparison of Clinical Outcomes Using “Elevate Anterior" versus "Perigee" System Devices for the Treatment of Pelvic Organ Prolapse. BioMed Res. Int. 2015, 2015, 479610. [CrossRef]

8. U.S. Food and Drug Administration. FDA Takes Action to Protect Women's Health, Orders Manufacturers of Surgical Mesh Intended for Transvaginal Repair of Pelvic Organ Prolapse to Stop Selling All Devices. Available online: https:/ / www.fda.gov/news-events/press-announcements/fda-takes-action-protect-womens-health-orders-manufacturerssurgical-mesh-intended-transvaginal (accessed on 8 January 2022).

9. Chang, T.-C.; Hsiao, S.-M.; Chen, C.-H.; Wu, W.-Y.; Lin, H.-H. Clinical Outcomes and Urodynamic Effects of Tailored Transvaginal Mesh Surgery for Pelvic Organ Prolapse. BioMed Res. Int. 2015, 2015, 191258. [CrossRef]

10. Zhu, L.; Lang, J.; Sun, Z.; Ren, C.; Liu, X.; Li, B. Pelvic reconstruction with mesh for advanced pelvic organ prolapse: A new economic surgical method. Menopause 2011, 18, 328-332. [CrossRef]

11. Nishizawa, O.; Kato, H.; Ishizuka, O. Advantages of self-tailored mesh for vaginal prolapse. Int. J. Urol. 2012, 19, 494-495. [CrossRef]

12. Gonocruz, S.G.; Hayashi, T.; Tokiwa, S.; Sawada, Y.; Okada, Y.; Yoshio, Y.; Krisna, R.; Kitagawa, Y.; Shimizu, Y.; Nomura, M. Transvaginal surgery using self-cut mesh for pelvic organ prolapse: 3-year clinical outcomes. Int. J. Urol. 2019, 26, 731-736. [CrossRef]

13. Rogowski, A.; Kluz, T.; Szafarowska, M.; Mierzejewski, P.; Sienkiewicz-Jarosz, H.; Samochowiec, J.; Bienkowski, P.; Baranowski, W. Efficacy and safety of the Calistar and Elevate anterior vaginal mesh procedures. Eur. J. Obstet. Gynecol. Reprod. Biol. 2019, 239, 30-34. [CrossRef]

14. Naumann, G.; Hüsch, T.; Mörgeli, C.; Kolterer, A.; Tunn, R. Mesh-augmented transvaginal repair of recurrent or complex anterior pelvic organ prolapse in accordance with the SCENIHR opinion. Int. Urogynecol. J. 2021, 32, 819-827. [CrossRef]

15. Mateu-Arrom, L.; Gutiérrez-Ruiz, C.; Palou Redorta, J.; Errando-Smet, C. Pelvic organ prolapse repair with mesh: Description of surgical technique using the Surelift ${ }^{\circledR}$ Anterior Repair System. Urol. Int. 2021, 105, 137-142. [CrossRef]

16. Skorupska, K.A.; Futyma, K.; Bogusiewicz, M.; Rechberger, E.; Ziętek-Strobl, A.; Miotła, P.; Wróbel, A.; Rechberger, T. Four-arm polypropylene mesh for vaginal vault prolapse-surgical technique and outcomes. Eur. J. Obstet. Gynecol. Reprod. Biol. 2020, 255, 203-210. [CrossRef]

17. Weintraub, A.Y.; Neuman, M.; Reuven, Y.; Neymeyer, J.; Marcus-Braun, N. Efficacy and safety of skeletonized mesh implants for advanced pelvic organ prolapse: 12-month follow-up. World J. Urol. 2016, 34, 1491-1498. [CrossRef]

18. Gon, L.M.; Riccetto, C.L.Z.; Neto, F.C.; Achermann, A.P.P.; Pereira, T.A.; Palma, P.C.R. Sacrospinous hysteropexy with an autologous rectus fascia sling for treatment of advanced apical pelvic organ prolapse. Int. Urogynecol. J. 2021, 32, 2291-2293. [CrossRef]

19. Chaus, F.M.; Funk, J.T.; Pangilinan, J.; Lin, F.C.; Twiss, C.O. Total Autologous Fascia Lata Anterior and Apical Pelvic Organ Prolapse Repair: A New Technique and Initial Experience. Urology 2020, 137, 190-195. [CrossRef]

20. Vasudeva, P.; Tyagi, V.; Kumar, N.; Yadav, S.; Prasad, V.; Iyer, S.G. "Mesh free” autologous transobturator mid urethral sling placement for predominant stress urinary incontinence: A pilot study. Neurourol. Urodyn. 2021, 40, 659-665. [CrossRef] 
21. Cormio, L.; Mancini, V.; Liuzzi, G.; Lucarelli, G.; Carrieri, G. Cystocele Repair by Autologous Rectus Fascia Graft: The Pubovaginal Cystocele Sling. J. Urol. 2015, 194, 721-727. [CrossRef]

22. Lo, T.-S.; Karim, N.B.; Cortes, E.F.M.; Wu, P.-Y.; Lin, Y.-H.; Tan, Y.L. Comparison between Elevate Anterior/Apical system and Perigee system in pelvic organ prolapse surgery: Clinical and sonographic outcomes. Int. Urogynecol. J. 2015, 26, 391-400. [CrossRef]

23. Vu, M.K.; Letko, J.; Jirschele, K.; Gafni-Kane, A.; Nguyen, A.; Du, H.; Goldberg, R.P. Minimal mesh repair for apical and anterior prolapse: Initial anatomical and subjective outcomes. Int. Urogynecol. J. 2012, 23, 1753-1761. [CrossRef]

24. Haylen, B.T.; De Ridder, D.; Freeman, R.M.; Swift, S.E.; Berghmans, B.; Lee, J.; Monga, A.; Petri, E.; Rizk, D.; Sand, P.K.; et al. An International Urogynecological Association (IUGA)/International Continence Society (ICS) joint report on the terminology for female pelvic floor dysfunction. Int. Urogynecol. J. 2010, 21, 5-26. [CrossRef]

25. Altman, D.G. Practical Statistics for Medical Research; Chapman \& Hall: London, UK, 1991; pp. $403-405$.

26. Kato, K.; Suzuki, S.; Ishiyama, A.; Kawanishi, H.; Matsui, H.; Kato, T.; Hirabayashi, H.; Hattori, R. Mesh exposure after transvaginal mesh prolapse surgery: Out of permissible range? Int. J. Urol. 2021, 28, 202-207. [CrossRef]

27. Vergeldt, T.F.M.; van Kuijk, S.; Notten, K.J.B.; Kluivers, K.B.; Weemhoff, M. Anatomical Cystocele Recurrence: Development and internal validation of a prediction model. Obstet Gynecol. 2016, 127, 341-347. [CrossRef]

28. Friedman, T.; Eslick, G.D.; Dietz, H.P. Risk factors for prolapse recurrence: Systematic review and meta-analysis. Int. Urogynecol. J. 2018, 29, 13-21. [CrossRef]

29. Long, C.-Y.; Lo, T.-S.; Wang, C.-L.; Wu, C.-H.; Liu, C.-M.; Su, J.-H. Risk factors of surgical failure following transvaginal mesh repair for the treatment of pelvic organ prolapse. Eur. J. Obstet. Gynecol. Reprod. Biol. 2012, 161, 224-227. [CrossRef]

30. Price, N.; Slack, A.; Jwarah, E.; Jackson, S. The incidence of reoperation for surgically treated pelvic organ prolapse: An 11-year experience. Menopause Int. 2008, 14, 145-148. [CrossRef]

31. Nüssler, E.; Eskildsen, J.K.; Bixo, M.; Löfgren, M. Impact of surgeon experience on routine prolapse operations. Int. Urogynecol. J. 2018, 29, 297-306. [CrossRef]

32. Khrucharoen, U.; Ramart, P.; Choi, J.; Kang, D.; Kim, J.-H.; Raz, S. Clinical predictors and risk factors for vaginal mesh extrusion. World J. Urol. 2018, 36, 299-304. [CrossRef]

33. Berglund, A.-L.; Eisemann, M.; Lalos, A.; Lalos, O. Social adjustment and spouse relationships among women with stress incontinence before and after surgical treatment. Soc. Sci. Med. 1996, 42, 1537-1544. [CrossRef]

34. Hsiao, S.-M.; Lin, H.-H. Impact of the mid-urethral sling for stress urinary incontinence on female sexual function and their partners' sexual activity. Taiwan J. Obstet. Gynecol. 2018, 57, 853-857. [CrossRef]

35. Huang, A.J.; Subak, L.L.; Thom, D.H.; Eeden, S.K.V.D.; Ragins, A.I.; Kuppermann, M.; Shen, H.; Brown, J.S. Sexual Function and Aging in Racially and Ethnically Diverse Women. J. Am. Geriatr. Soc. 2009, 57, 1362-1368. [CrossRef]

36. Ehsani, N.; Ghafar, M.A.; Antosh, D.D.; Tan-Kim, J.; Warner, W.B.; Mamik, M.M.; Brown, H.; Chung, C.P.; Segal, S.; Abed, H.; et al. Risk Factors for Mesh Extrusion After Prolapse Surgery: A case-control study. Female Pelvic Med. Reconstr. Surg. 2012, 18, 357-361. [CrossRef]

37. Kawaguchi, S.; Narimoto, K.; Urata, S.; Takeyama, M.; Kadono, Y.; Mizokami, A. Predictors of persistent stress urinary incontinence after transvaginal mesh repair. BMC Womens Health 2018, 18, 174. [CrossRef]

38. Seki, N.; Shahab, N.; Hara, R.; Takei, M.; Yamaguchi, A.; Naito, S. Voiding dynamics in women with stress urinary incontinence and high-stage cystocele. Int. J. Urol. 2011, 18, 219-224. [CrossRef]

39. Kitagawa, Y.; Narimoto, K.; Urata, S.; Kawaguchi, S.; Kuribayashi, M.; Namiki, M. High urinary flow in women with stress incontinence: Corrected flow-age nomogram evaluation after a transobturator tape procedure. Int. Urogynecol. J. 2016, 27, 1075-1080. [CrossRef]

40. Pilsetniece, Z.; Vjaters, E. The role of conventional urodynamic in diagnosing specific types of urinary incontinence in women. Turk. J. Urol. 2020, 46, 134-139. [CrossRef]

41. de Boer, T.A.; Kluivers, K.B.; Withagen, M.I.J.; Milani, A.L.; Vierhout, M.E. Predictive factors for overactive bladder symptoms after pelvic organ prolapse surgery. Int. Urogynecol. J. 2010, 21, 1143-1149. [CrossRef]

42. de Boer, T.A.; Vierhout, M.E. Predictors for overactive bladder symptoms after pelvic organ prolapse surgery. Curr. Opin. Obstet. Gynecol. 2011, 23, 366-370. [CrossRef]

43. Tomoe, H. Improvement of overactive bladder symptoms after tension-free vaginal mesh operation in women with pelvic organ prolapse: Correlation with preoperative urodynamic findings. Int. J. Urol. 2015, 22, 577-580. [CrossRef] [PubMed]

44. Foster, R.; Barber, M.D.; Parasio, M.F.R.; Walters, M.D.; Weidner, A.C.; Amundsen, C.L. A prospective assessment of overactive bladder symptoms in a cohort of elderly women who underwent transvaginal surgery for advanced pelvic organ prolapse. Am. J. Obstet. Gynecol. 2007, 197, 82.e1-82.e4. [CrossRef] [PubMed]

45. Ugianskiene, A.; Kjærgaard, N.; Larsen, T.; Glavind, K. What happens to urinary incontinence after pelvic organ prolapse surgery? Int. Urogynecol. J. 2019, 30, 1147-1152. [CrossRef] [PubMed] 